\title{
The effect of post-growth rapid thermal annealing on InAs/InGaAs dot-in-a-well structure monolithically grown on $\mathrm{Si}$
}

Cite as: J. Appl. Phys. 125, 135301 (2019); https://doi.org/10.1063/1.5085175

Submitted: 10 December 2018 . Accepted: 10 March 2019 . Published Online: 01 April 2019

W. Li, S. Chen (D), J. Wu (D), A. Li, M. Tang (D), L. Yang, Y. Chen, A. Seeds, H. Liu, and I. Ross
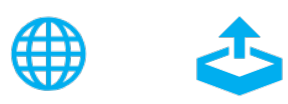

\section{ARTICLES YOU MAY BE INTERESTED IN}

$\mathrm{ZnO} /(\mathrm{Zn}) \mathrm{MgO}$ polar and nonpolar superlattices

Journal of Applied Physics 125, 135702 (2019); https://doi.org/10.1063/1.5085055

Direct and indirect optical transitions in bulk and atomically thin $\mathrm{MoS}_{2}$ studied by photoreflectance and photoacoustic spectroscopy

Journal of Applied Physics 125, 135701 (2019); https://doi.org/10.1063/1.5080300

Myths about new ultrahard phases: Why materials that are significantly superior to diamond in elastic moduli and hardness are impossible

Journal of Applied Physics 125, 130901 (2019); https://doi.org/10.1063/1.5082739

Applied Physics Reviews Now accepting original research

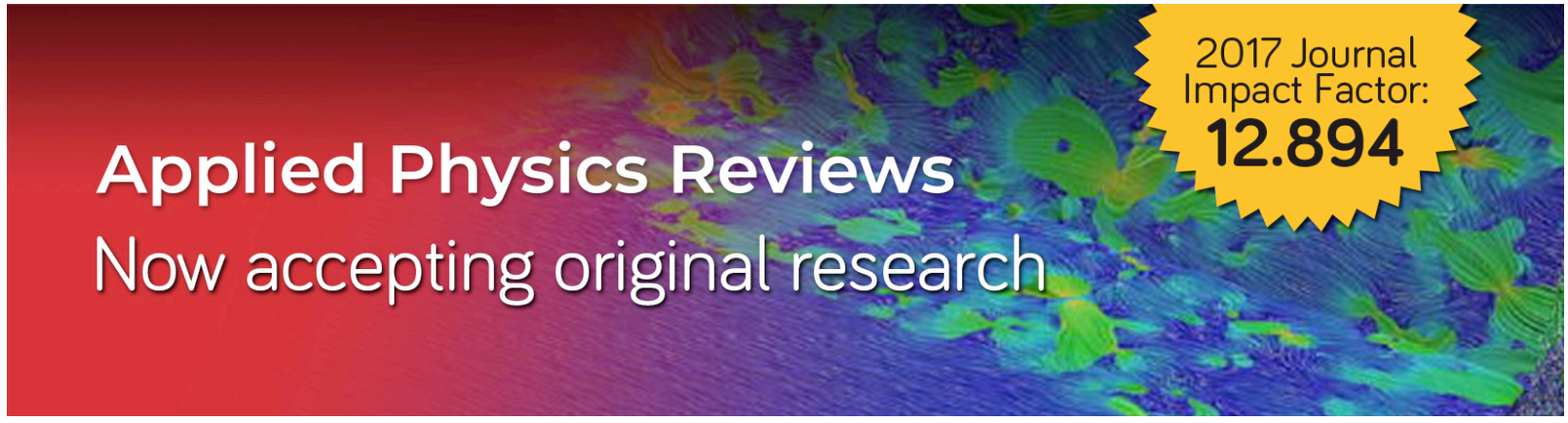




\title{
The effect of post-growth rapid thermal annealing on InAs/InGaAs dot-in-a-well structure monolithically grown on $\mathrm{Si}$
}

\author{
Cite as: J. Appl. Phys. 125, 135301 (2019); doi: 10.1063/1.5085175 \\ Submitted: 10 December 2018 - Accepted: 10 March 2019 . \\ Published Online: 1 April 2019
}

\section{W. Li, ${ }^{1,2}$ S. Chen, ${ }^{3, a)}$ (D) J. Wu, ${ }^{3}$ (D) A. Li, ${ }^{7}$ M. Tang, ${ }^{3}$ (D) L. Yang, ${ }^{7}$ Y. Chen, ${ }^{7}$ A. Seeds, ${ }^{3}$ H. Liu, ${ }^{3}$ and I. Ross ${ }^{2}$}

\author{
AFFILIATIONS \\ ${ }^{1}$ Institute of Microstructure and Properties of Advanced Materials, Beijing University of Technology, Beijing 100124, China \\ ${ }^{2}$ Department of Materials Science and Engineering, University of Sheffield, Sheffield S3 7HQ, United Kingdom \\ ${ }^{3}$ Department of Electronic and Electrical Engineering, University College London, London WC1E 7JE, United Kingdom \\ a)Siming.chen@ucl.ac.uk
}

\begin{abstract}
The effect of post-growth annealing (PGA) on dot-in-well (DWELL) structures grown on Si substrates has been studied. The photoluminescence (PL) measurements showed that, compared to the DWELL structures directly grown on GaAs, the PGA process induces a distinct difference in the tuning of the emission properties. Then, transmission electron microscopy imaging of the samples revealed that PGA improved the uniformity of quantum dots (QDs) while the size of the QDs increased, in agreement with a corresponding red shift and a decrease of the full width at half maximum in the PL emission spectrum. Finally, energy-dispersive $\mathrm{x}$-ray linescan provided a quantitative analysis of the composition change of DWELL grown on $\mathrm{Si}$ in the as-grown, $700^{\circ} \mathrm{C}$ annealed, and $800^{\circ} \mathrm{C}$ annealed samples. The change in the $\mathrm{In}_{\mathrm{L}} / \mathrm{Ga}_{\mathrm{K}} \mathrm{concentration}$ ratio became gradual between the QDs and surrounding materials after $800^{\circ} \mathrm{C}$ annealing. The analysis of the optical properties, morphology evolution, and compositional change of the QDs as a function of the annealing temperature showed good agreement.
\end{abstract}

Published under license by AIP Publishing. https://doi.org/10.1063/1.5085175

\section{INTRODUCTION}

Compared with the traditional re-growth and selective area growth technique, rapid thermal annealing (RTA) provides a low-cost, simple, and powerful way to tune and enhance the structural and optical qualities of nanostructures, such as the bandgap energy, confinement, and absorption, and offers a potential route to develop new types of devices and integrated optoelectronic circuits. ${ }^{1}$ For example, Sengupta et al. ${ }^{2}$ reported tuning of the detection wavelength of infrared photodetectors, Zhang et al. ${ }^{3}$ showed an improvement on the bandwidth of superluminescent diodes, while Tatebayashi et al. ${ }^{4}$ and $\mathrm{Li}$ et $a .^{5}$ demonstrated the control of the lasing wavelength of quantum dot (QD) lasers. In addition, rapid thermal annealing has been used to reduce the defects generated by low-temperature growth $^{6}$ or lattice mismatch. ${ }^{7}$ On the other hand, there is evidence to suggest that high-temperature thermal annealing will potentially influence the shape, size, and composition of quantum-confined semiconductor structures due to the intermixing process. ${ }^{8-12}$

Many techniques have been applied to deliberately promote intermixing-for example, impurity-induced disordering, impurity-free vacancy disordering, etc. ${ }^{13,14}$ In a typical GaAs system, a $\mathrm{SiO}_{2}$ cap is widely used during the annealing process to induce $\mathrm{Ga}$ atoms to dissolve into the $\mathrm{SiO}_{2}$ cap and create $\mathrm{Ga}$ vacancies which then diffuse through the heteroepitaxial structure and cause intermixing. ${ }^{15}$ The process of intermixing provides an attractive means to combine subtle bandgap tuning with the benefits already offered by QD technology. During the quantum dot intermixing (QDI) process, the atomic inter-diffusion between the QDs and the surrounding barrier layers is not only influenced by the difference in thermal expansion coefficients between the QDs and the surrounding materials, giving rise to $\mathrm{In} / \mathrm{Ga}$ inter-diffusion, but is also strongly affected by the size, shape, and strain distribution within and around the QD. ${ }^{14}$ The change in the optical properties and the related evolution of the QD structure has been presented by a number of previous researchers. In general, QDI caused by In/Ga intermixing between QDs and surrounding materials leads to a change in the composition and size of the QDs and also leads to a change in the QDs confining potential. There are generally three main effects on the optical properties of QDs: a blue shift of the PL 
spectrum, a reduction or increase in the FWHM of the QD ensemble emission (broadening emission from the QD ensemble at a relatively low temperature of $\sim 650^{\circ} \mathrm{C}$ for highly uniform QDs), ${ }^{16,17}$ and a reduction in the inter-sublevel energy spacing between confined states..$^{8-12}$

Even though the process of PGA has been documented for QDs and other structures grown on GaAs substrates by several groups, ${ }^{2,4,10,11}$ a similar annealing study of QDs grown on $\mathrm{Si}$ is still to be undertaken. Hence, in this current work, systematic studies on the effect of rapid thermal annealing on the optical properties of QDs grown monolithically on $\mathrm{Si}$ are performed. There have been promising results obtained from post-growth thermal annealing related to the inter-diffusion induced tuning of materials and devices. ${ }^{2,4,8,18-20}$ For QDs grown on GaAs, the influence of thermal annealing mainly involves intermixing and shape change. However, for QDs grown on $\mathrm{Si}$, the issue becomes more complicated. The complications come from the high density of defects generated in the buffer layers due to the large lattice mismatch between III-V compounds and $\mathrm{Si}$ and the residual stress induced by the mismatch of the thermal expansion coefficients of these two materials. Despite these difficulties, it is nevertheless worthwhile to investigate the effects of post-growth thermal annealing on these materials to enable a more robust comparison between III-V on Si and the conventional process. The motivation here is that current epitaxially grown III-V-on-Si materials are far inferior to the III-V-on-GaAs materials in terms of total defect density while post-growth annealing has successfully been used to reduce the defect density by about two orders in epitaxially grown GaAs-on-Si.

\section{EXPERIMENTAL DETAILS}

\section{A. Sample growth}

The growth details of the InAs/InGaAs dot-in-well (DWELL) structure monolithically grown on Si substrates have been shown by Wang et al. ${ }^{21,22}$ The schematic sample structure and crosssection bright field scanning transmission electron microscopy (BF-STEM) image of the as-grown sample are shown in Figs. 1(a) and $1(\mathrm{~b})$, respectively.

\section{B. Sample preparation}

Before the thermal annealing process, the whole wafer was first capped with a layer of a plasma-enhanced chemical-vapour deposited (PECVD) $\mathrm{SiO}_{2}$ film. Then, the wafer was cleaved to several pieces. Next, the rapid thermal annealing treatment was carried out on different pieces of sample at $650^{\circ} \mathrm{C}, 700{ }^{\circ} \mathrm{C}, 750^{\circ} \mathrm{C}$, and $800^{\circ} \mathrm{C}$ for $30 \mathrm{~s}$ under nitrogen conditions.

Cross-sectional samples for TEM and STEM imaging were prepared parallel to the $[01 \overline{1}]$ plane by conventional grinding and mechanical polishing methods, followed by Ar-ion milling to electron transparency.

\section{Characterization methods}

For optical studies, the $\mathrm{SiO}_{2}$ capping layer was first removed by hydrogen fluoride treatment. PL measurements were performed in a closed-cycle He cryostat under excitation from a $532 \mathrm{~nm}$ solidstate laser. The signal was dispersed by a monochromator and

collected using a germanium detector. The laser spot was unfocused. Samples of the DWELL structure grown on GaAs with the same layers were processed using identical conditions for comparison.

A series of initial BF-STEM images were obtained using a C-FEG JEOL R005 double aberration corrected TEM/STEM operating at $300 \mathrm{kV}$. Lattice-resolved STEM high angle annular dark field (HAADF) Z-contrast images were then obtained with a convergence semi-angle of 21 mrads and a STEM inner annular collection angle of 62 mrads. HAADF-STEM images of the as-grown and annealed specimens were obtained at $\sim 600 \mathrm{kX}$ for size distribution analysis. Intensity distribution lines parallel/perpendicular to the growth plane have been drawn across the QDs in the HAADF-STEM images so that the interfaces of QDs are easy to define.

Composition measurements were performed using a JEOL 2010F microscope in STEM mode equipped with an energy-dispersive x-ray (EDX) analyzer controlled by the Oxford Instruments LINK ISIS300 software. Medium spot size and an $\sim 9.5 \mathrm{mrad}$ collection semi-angle were used with an estimated probe diameter of $0.4 \mathrm{~nm}^{23}$ Based on the thickness calculated by electron energy loss spectroscopy (EELS), the beam broadening was estimated to be around $4.5-8 \mathrm{~nm}$. Therefore, EDX linescans were performed across QDs parallel to the growth plane by taking the EDX spectrum from 30 points along

(a)

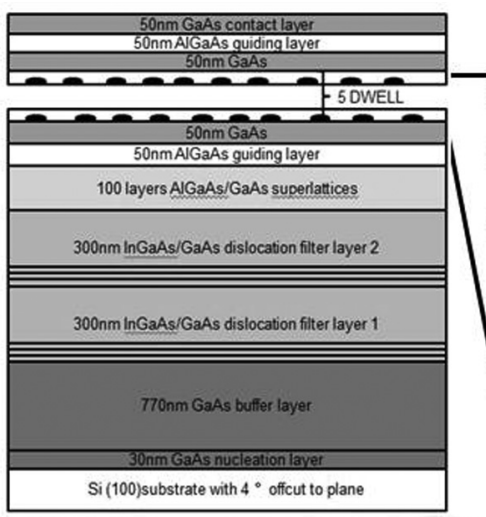

(b)

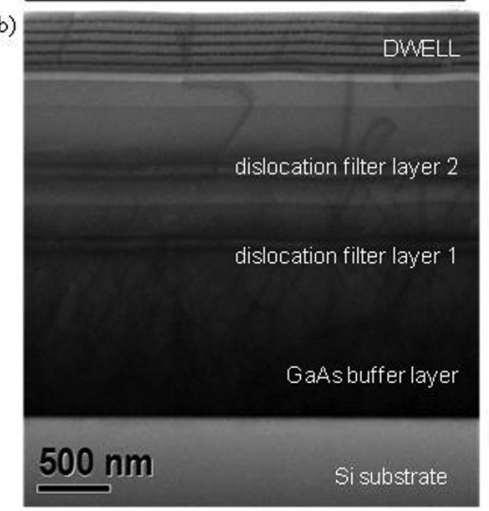

FIG. 1. (a) Schematic of the InAs/lnGaAs DWELL structure monolithically grown on $\mathrm{Si}$ substrates. The inset sketches the details of DWELL layers. (b) Cross-section BF-STEM image of the as-grown structure. 
$145 \pm 10 \mathrm{~nm}$. The acquisition live time for taking each point spectrum was set to $20 \mathrm{~s}$.

\section{RESULTS AND DISCUSSION}

\section{A. Photoluminescence of QDs}

Figure 2 shows the normalized PL spectra of the samples grown on $\mathrm{Si}$ and $\mathrm{GaAs}$ substrates, respectively. For both sets of samples, no significant spectrum shift is observed until an annealing temperature of $700{ }^{\circ} \mathrm{C}$. However, a distinct blue shift is observed for the PL spectra as the annealing temperatures are increased above $700^{\circ} \mathrm{C}$.

This shift is attributed to the In-Ga intermixing during the rapid thermal annealing treatment. The relative difference in the shift for the samples grown on $\mathrm{Si}$ and GaAs substrates is small. As we expect that the residual stress in the active region to be small, the influence of residual stress on the intermixing should also be small.

Figure 3 shows the integrated PL intensity of the samples grown on $\mathrm{GaAs}$ and $\mathrm{Si}$ as a function of annealing temperatures. At $650{ }^{\circ} \mathrm{C}$, the emission signals for both $\mathrm{GaAs}$ and Si-based samples are improved. At $700^{\circ} \mathrm{C}$, the sample grown on Si starts to show a reduction in PL intensity. However, the sample grown on GaAs does not show an emission reduction until $750^{\circ} \mathrm{C}$. Figure 3 also compares the emission intensity of the samples grown on $\mathrm{GaAs}$ and $\mathrm{Si}$ substrates. It is clearly shown that the annealing has a more positive impact on the DWELL grown on GaAs. The change in the room temperature emission intensity upon thermal annealing mainly comes from the change in the non-radiative recombination rate and change in the thermal-assisted escaping rate. By comparing the different influence of thermal annealing on the PL of the samples grown on GaAs and $\mathrm{Si}$, the effects of annealing on the structural microstructure are quite different for each case. From previous studies on rapid thermal annealing of epitaxial GaAs on $\mathrm{Si}$, the thermal annealing can improve the structural quality of $\mathrm{GaAs}$ on $\mathrm{Si}$; however, the post-growth thermal annealing tends to generate high-density deep levels induced by increased stress in the material, which can explain the more distinct improvement in photoluminescence emission after annealing for the sample grown on GaAs. In addition, modification of the confinement may also play a role in the difference observed in Fig. 3 for the samples grown on $\mathrm{GaAs}$ and $\mathrm{Si}$. The intermixing is a thermally activated process. The defect density and residual stress can both affect the intermixing which then results in a different confinement and then a different thermally assisted escape rate.

To obtain further insight into the annealing effects on the optical properties of the DWELL structures, the blue shift of the two sets of samples were compared for different annealing temperatures as shown in Fig. 4. At relatively low annealing temperatures, the shift of the sample on Si is larger. On the other hand, the shift of the sample grown on $\mathrm{GaAs}$ becomes larger as the annealing temperature increases over $750{ }^{\circ} \mathrm{C}$. Therefore, the rapid thermal annealing indeed has different degrees of influences on intermixing for the samples grown on GaAs and Si. More interestingly, the blue shift in the emission peak results from a change in the dot (a)

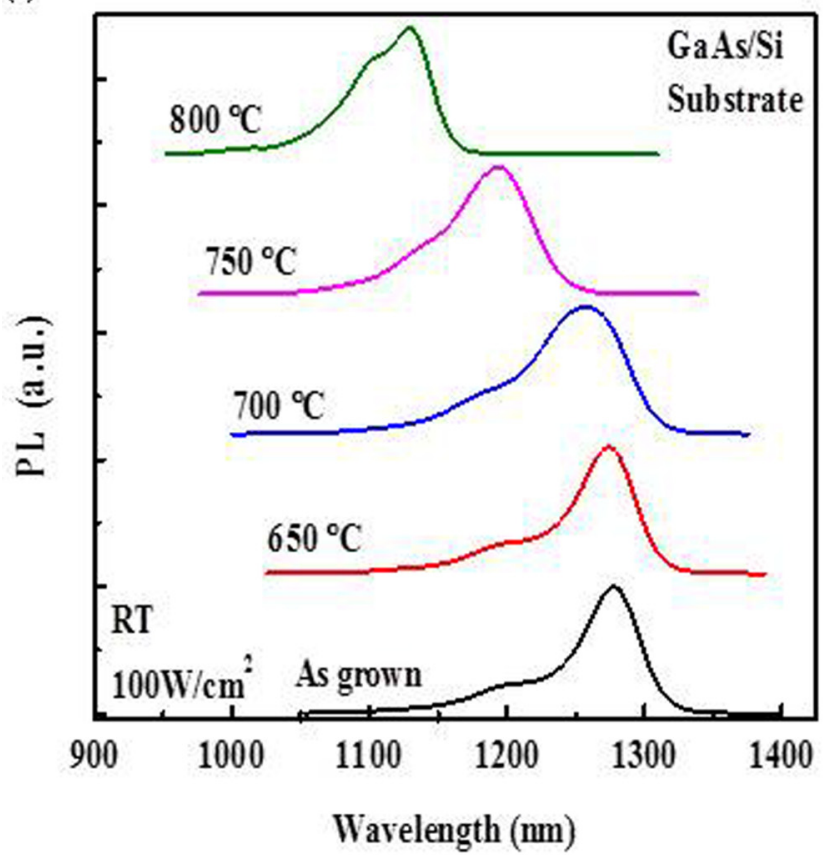

(b)

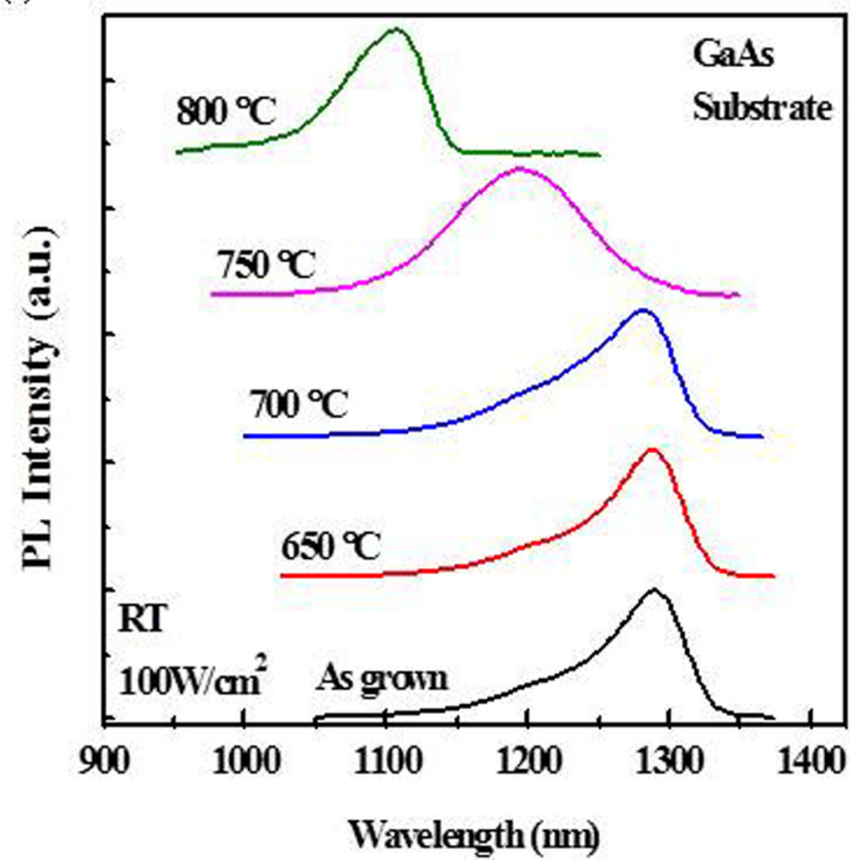

FIG. 2. Normalized PL spectra of the InAs/InGaAs DWELL structure grown on the (a) Si substrate and (b) GaAs substrate after being annealed at different temperatures. The laser excitation is $\sim 100 \mathrm{~W} / \mathrm{cm}^{2}$. 
(a)

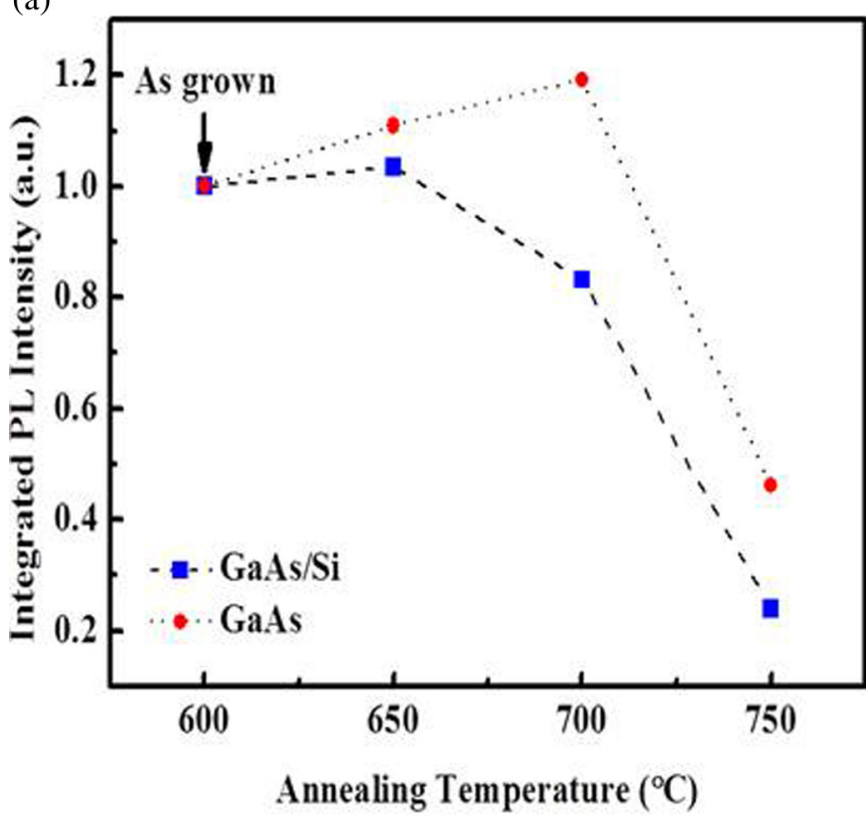

(b)

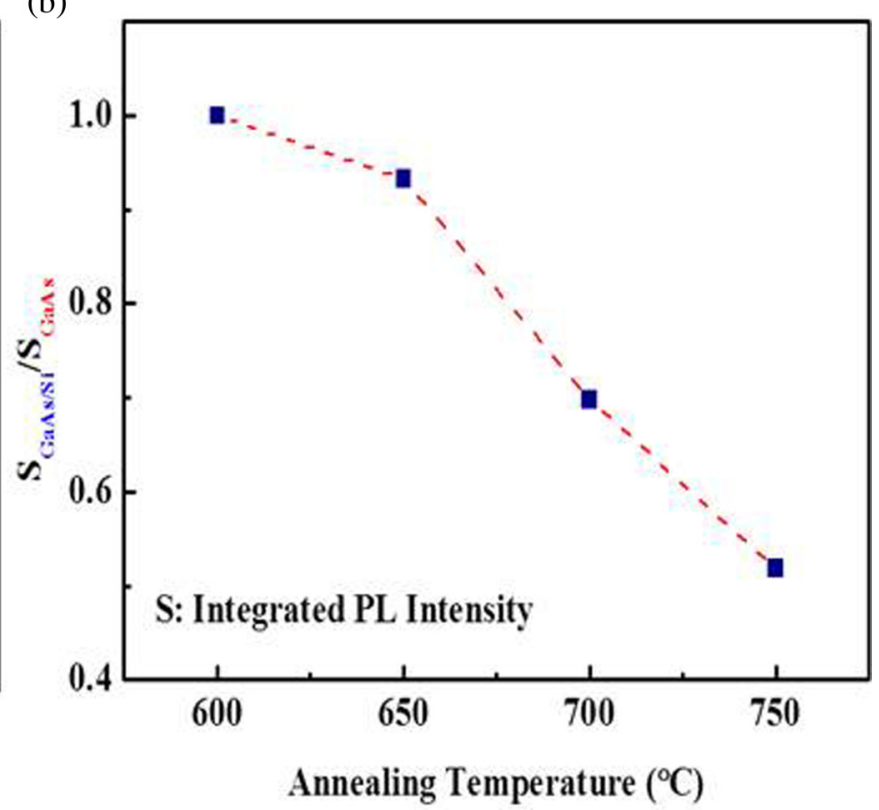

FIG. 3. (a) Integrated PL intensity of the annealed samples normalized to the as-grown sample. (b) The ratio between the integrated PL intensity of DWELLs on GaAs and $\mathrm{Si}$ substrates at different annealing temperatures.

composition in the shallower confining potential. In addition, an effective incensement in the dot size during inter-diffusion results in a reduced size distribution and hence a smaller FWHM of the emission peak. $^{11,24}$

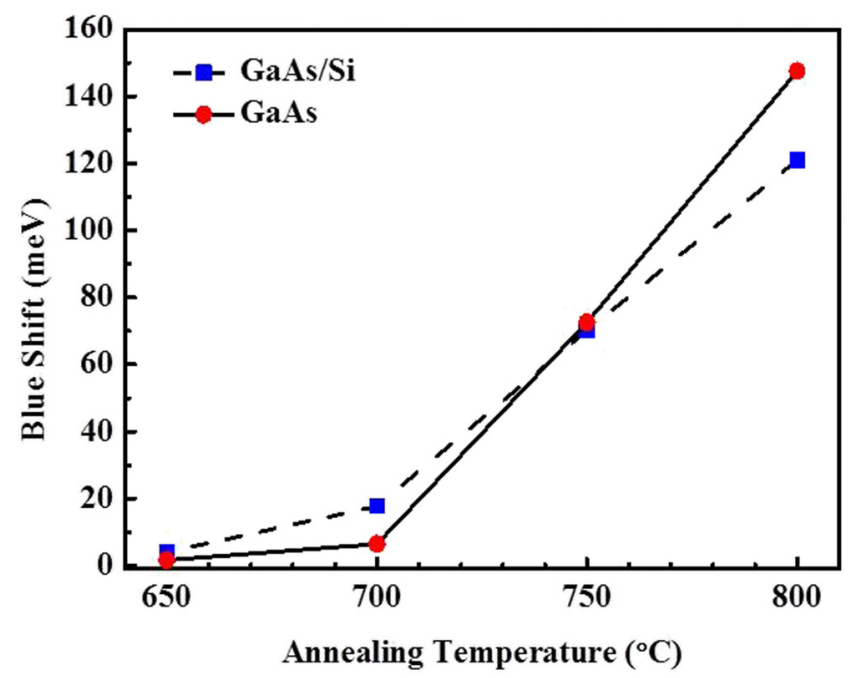

FIG. 4. PL emission peak as a function of annealing temperature.
Figure 5 shows the linewidth of the PL peak emission as a function of temperature. Regarding the measured linewidth which is the FWHM of the line that corresponds to the best fit assuming the sum of Gaussian functions [an example of how FWHM being determined is further illustrated in Fig. 6(a)], the FWHM is observed to increase for both the samples grown on $\mathrm{GaAs}$ and $\mathrm{Si}$ substrates. The increase in FWHM is as large as $50 \mathrm{~nm}$ for the DWELL sample grown on GaAs after being annealed at $750{ }^{\circ} \mathrm{C}$. The maximum increase in FWHM for the sample on $\mathrm{Si}$ is smaller, about $20 \mathrm{~nm}$, and is obtained at a lower temperature. A further increase in the annealing temperature results in shrinkage in the spectral width. There is a notable correlation between the blue shift and the change in FWHM of the samples. In general, for annealing at higher temperatures, the size distribution of the QDs will be greatly improved via the process of In-Ga intermixing ${ }^{7-11}$ owing to the increase in dot size and the reduction in composition fluctuations. Therefore, we presume that the thermal annealing-induced intermixing on $\mathrm{Si}$ substrates is faster, which leads to a more distinct change of emission peak and linewidth at lower temperatures. At high temperature, the influence of annealing on the size distribution is more dominant. As a result, the FWHM reduces again, and the blue shift of the DWELL on GaAs becomes larger than that of the sample on $\mathrm{Si}$ due to the smaller change in size and higher quantum confinement.

Figure 6(a) shows an example of a Gaussian fit of PL spectra from the GaAs grown on Si. A nearly perfect fit with three Gaussians for the PL spectra is observed. The green dashed line 


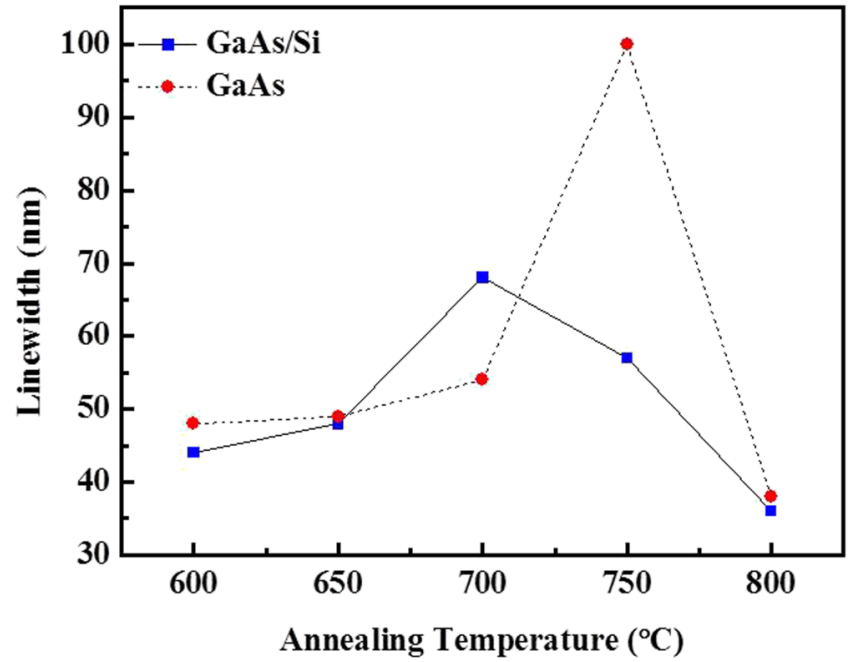

FIG. 5. FWHM of the PL emission peaks as a function of annealing temperatures for both the samples grown on GaAs and Si substrates.

peak is for ground state emission, the pink dashed line is for first excited state emission, and the blue dashed line is for the second excited state. Then, $\Delta E$ denotes the separation between the ground and first excited state as shown in Fig. 6(a). The energetic positions of the ground and excited state emissions were determined for each spectrum for the QDs grown on $\mathrm{Si}$ substrates. For the sample annealed at $800^{\circ} \mathrm{C}$, no separation was observed as the two peaks merged into a single peak. The spacing between the ground and excited state emission as a function of annealed temperature for QDs grown on Si substrates is shown in Fig. 6(b). By increasing the annealing temperature, the separation between the ground and first excited state emission reduced gradually from $73 \mathrm{~nm}$ in the as-grown sample to $47 \mathrm{~nm}$ in the sample annealed at $750{ }^{\circ} \mathrm{C}$ which agrees with the previous observations. ${ }^{24-26}$ The rate of reduction also increased as the annealing temperature increased. Some theoretical studies $^{27-29}$ have predicted that the space between the ground state and the excited state of InAs/GaAs QDs decreases as the aspect ratio between the height $h$ and the base length $b$ decreases.

All these assumptions are based on the observations from the PL measurements. TEM and related measurements are required to fully understand the impact of rapid thermal annealing on III-Vs epitaxially grown on $\mathrm{Si}$.

\section{B. Size analysis of QDs}

BF-STEM images of as-grown and annealed specimens were obtained at relatively low magnification. Figure 7 shows the BF-STEM images of DWELL of (a) the as-grown sample, (b) the $700{ }^{\circ} \mathrm{C}$ annealed sample, and (c) $800^{\circ} \mathrm{C}$ annealed sample and a corresponding schematic diagram of the morphological evolution. As the images show, QDs in the as-grown sample exhibit a sharp interface and inhomogeneous dot size. After $700^{\circ} \mathrm{C}$ annealing, the interface of QDs and the surrounding matrix became less distinct possibly due to the thermal intermixing process. However, after $800^{\circ} \mathrm{C}$ annealing, the QDs uniformity had significantly improved with an increase in the average dot size, with all the dots appearing "flatter." (a)

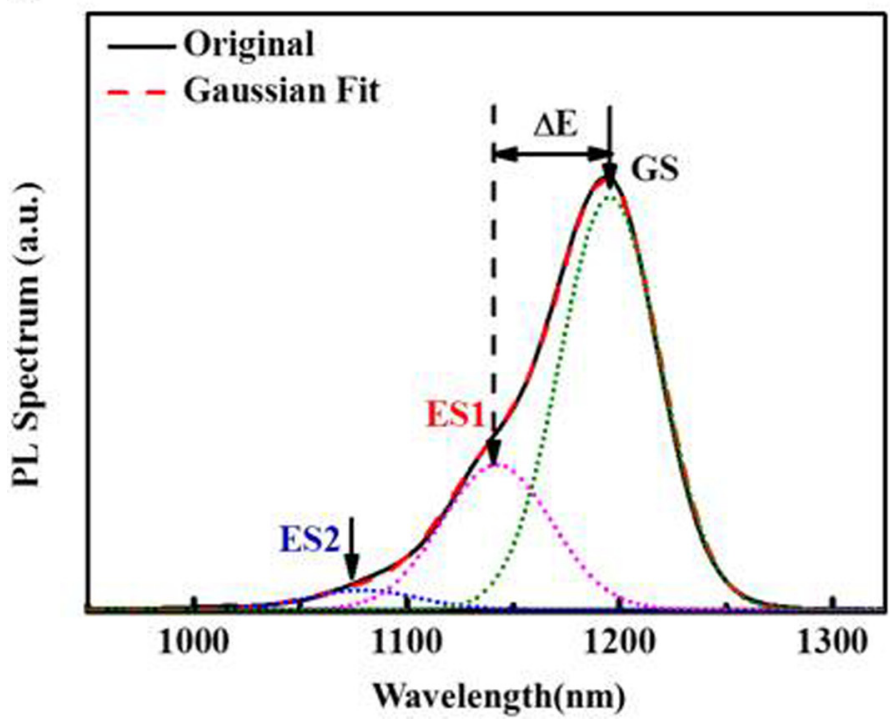

(b)

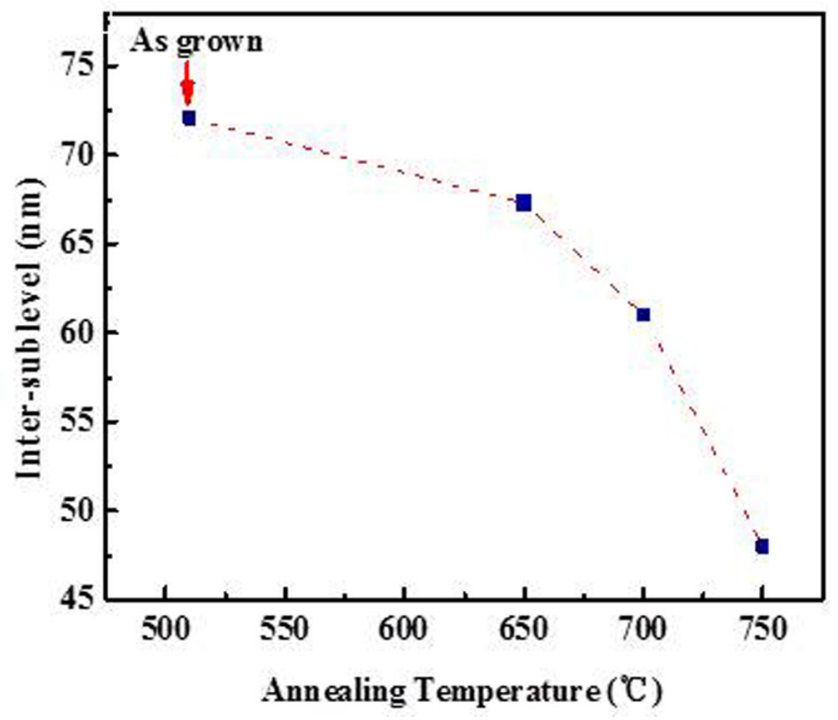

FIG. 6. (a) The peak position of ground and excited state emissions was determined by Gaussian fit. (b) The separation between the GS and ES1 as a function of annealing temperature for QDs grown on Si substrates. 

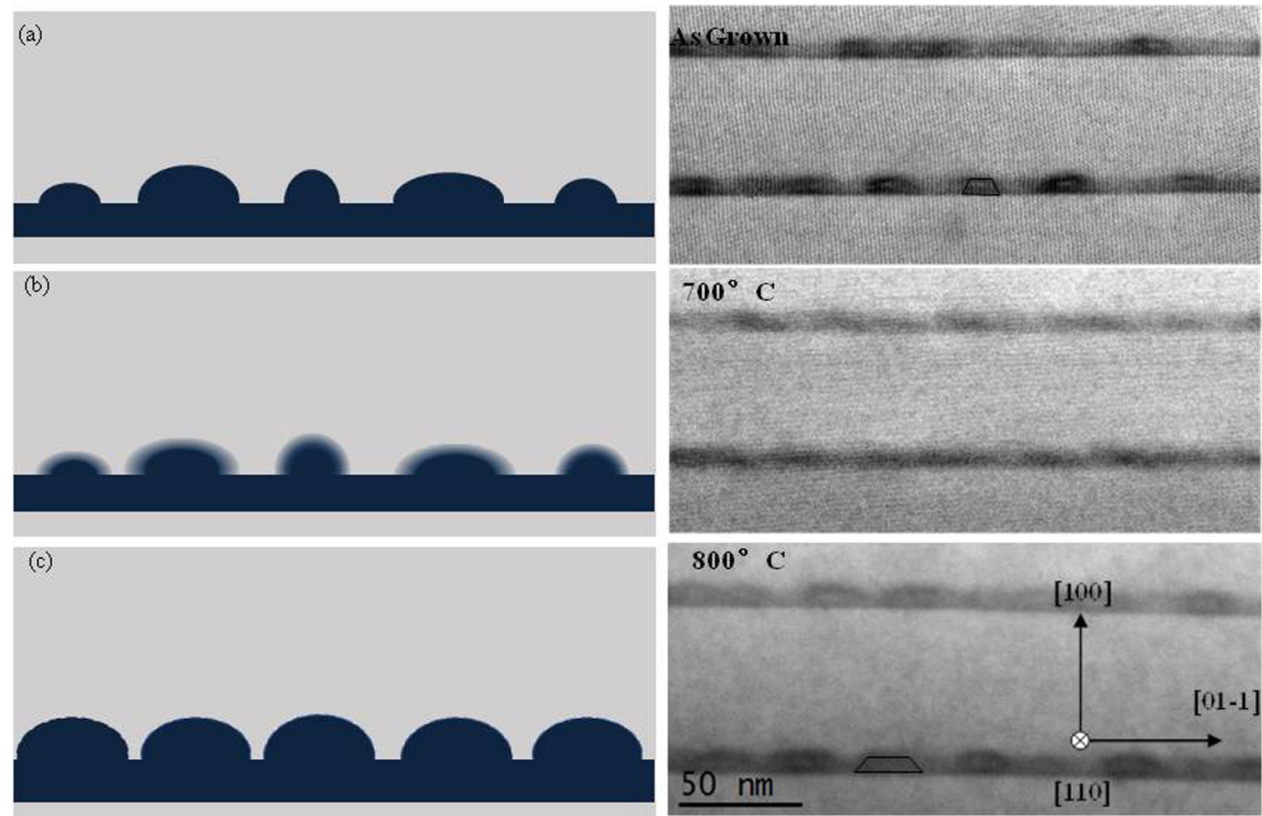

FIG. 7. $\mathrm{C}_{\mathrm{s}}$-corrected BF-STEM images of DWELL as a function of annealed temperature and related schematic diagram.
Approximately 100 QDs have been measured for each of the as-grown, $700{ }^{\circ} \mathrm{C}$ annealed, and $800^{\circ} \mathrm{C}$ annealed samples using HAADF imaging at $600 \mathrm{k}$ magnification to determine the width and height of the QDs. The widths and heights of the QDs were measured by drawing intensity line profiles across the HAADF images. The widths and heights of the QDs in the as-grown sample were $28.3 \pm 4.6 \mathrm{~nm}$. The widths of the QDs in $700^{\circ} \mathrm{C}$ and $800^{\circ} \mathrm{C}$ annealed samples increase to $30 \pm 4 \mathrm{~nm}$ and $36.4 \pm 2.9 \mathrm{~nm}$, respectively. On the other hand, the heights do not appear to change significantly, when measurement error is taken into consideration. This would suggest that the inter-diffusion predominates within the (100) plane rather than the [100] growth direction. Considering the theoretical QD geometries proposed in Refs. 26-28, this means the aspect ratio between the height $h$ and the base length $b$ is smaller after PGA which is in agreement with the PL observations.

As the image contrast in the HAADF image is approximately proportional to the square of the mean atomic number, assuming pure Rutherford scattering, such images offer an insight into the relative local chemistry of the features. Figure 8 shows a series of lattice-resolved HAADF-STEM Z-contrast images of typical single QDs as a function of the annealing temperature. The increased contrast brightness in the region of the QD reflects the higher mean atomic number due to the presence of indium. While the Z-contrast images are not specifically indium concentration maps, they highlight the changes in the QD morphology which is likely to be associated with localized indium diffusion. The dot width is $8.8 \pm 1 \mathrm{~nm}$ in the as-grown dots. After $700{ }^{\circ} \mathrm{C}$ annealing, the image contrast becomes more uniform across the dot region, and the QD size increased laterally. After $800^{\circ} \mathrm{C}$ annealing, the lateral dot size further increased. It is important to note that although the overall aspect ratio appears to change as a function of the annealing temperature, the basic shape morphology of QDs remains essentially the same, which is in agreement with the observation proposed in Fig. 7.

\section{EDX analysis of QDs}

Quantitative chemical concentrations of In and $\mathrm{Ga}$ in the InGaAs DWELL samples could be derived by the application of thickness dependent $k$-factors, taking As (the group V element) as a reference at an assumed concentration of $50 \%$. The process of annealing of the InGaAs alloy system is group III sub-lattice $(\mathrm{In} / \mathrm{Ga})$ inter-diffusion predominates. As the peak fitting and built-in $k$-factors were unavailable above $20 \mathrm{kV}$ energy in the ISIS300 software, the In K-lines at $24-27 \mathrm{kV}$ were unavailable for analysis, and therefore compositional analysis makes use of the In L-lines. Then, the effective $k$-factors of $\mathrm{In}_{\mathrm{L}}$ in respect to $\mathrm{Ga}_{\mathrm{K}}$ are used which is given by

$$
k_{I n L, G a K}^{*}=\frac{x I_{G a K} A_{I n}}{(1-x) I_{I n L} A_{G a}},
$$

in which $x$ is the indium fraction of $\operatorname{In}_{\mathrm{x}} \mathrm{Ga}_{1-\mathrm{x}} \mathrm{As}$, which is nominally 0.3 in our samples; $I$ is the integrated intensity of the $\mathrm{x}$-ray spectrum; and $A$ is the atomic weight of the elements $\left(A_{\mathrm{As}}=74.9\right.$, $A_{\mathrm{Ga}}=69.7$, and $A_{\mathrm{In}}=114.8$ ).

Parri et al..$^{30}$ and Walther and Wang ${ }^{31}$ have performed CASINO simulations of the $\mathrm{K} / \mathrm{L}$ line intensity ratios vs sample thickness and the $k_{I n L, G a K}^{*}$ vs K/L ratios with different In concentrations $(x)$ and have plotted, and linear least-squares regressions of these data have been calculated. The thickness of the region under analysis in the as-grown, $700^{\circ} \mathrm{C}$ annealed, and $800^{\circ} \mathrm{C}$ annealed samples was measured by EELS to be $103 \pm 15 \mathrm{~nm}, 60 \pm 12.5 \mathrm{~nm}$, and $85 \pm 14 \mathrm{~nm}$, respectively. Therefore, the $k$-factor, $k_{I n L, G a K}^{*}$, 


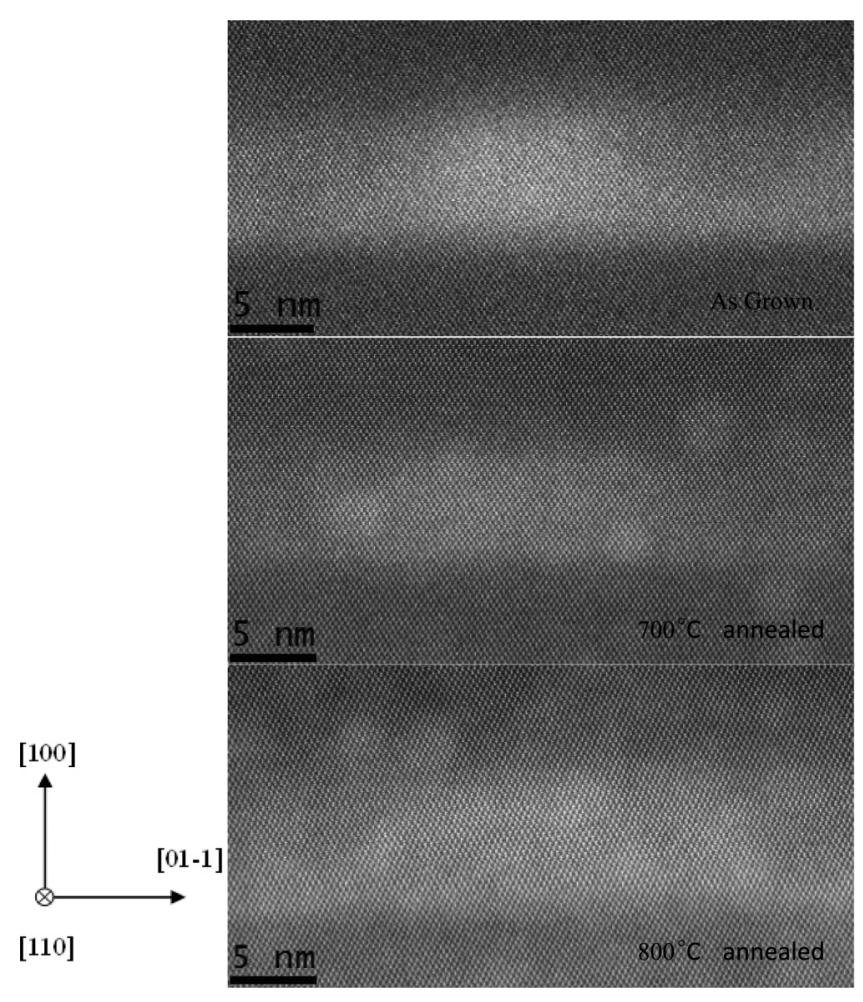

FIG. 8. $\mathrm{C}_{\mathrm{s}}$-corrected high-resolution HAADF-STEM images of a single $\mathrm{QD}$ as a function of annealed temperature.

can be taken by substituting the thickness into the two linear least-squares regression lines, ${ }^{30,31}$ directly. Finally, based on the Cliff-Lorimer equation

$$
\frac{C_{I n L}}{C_{G a K}}=k_{I n L, G a K}^{*} \frac{I_{I n L}}{I_{G a K}} .
$$

The resulting apparent concentration ratio of $\operatorname{In}_{\mathrm{L}}$ and $\mathrm{Ga}_{\mathrm{K}}$ plots is shown in Fig. 9(d). The position of the QDs through the plot of the concentration ratio of $\mathrm{In}_{\mathrm{L}} / \mathrm{Ga}_{\mathrm{K}}$ of the as-grown sample is clearly defined. After $700{ }^{\circ} \mathrm{C}$ annealing, the $\mathrm{In}_{\mathrm{L}} / \mathrm{Ga}_{\mathrm{K}}$ concentration ratio became rambling, which is consistent with the poorly defined QDs interface shown in Fig. 7. This phenomenon may be caused by the fact that the Ga vacancy diffusion coefficient is constant and independent of the In composition at relatively low-temperature annealing. ${ }^{32}$ In other words, it may be caused by the Ga-in diffusion rate being different from the In-out diffusion rate. After $800{ }^{\circ} \mathrm{C}$ annealing, the $\mathrm{In}_{\mathrm{L}} / \mathrm{Ga}_{\mathrm{K}}$ concentration ratio of each QD appeared as a Gaussian distribution, and the trend of the $\mathrm{In}_{\mathrm{L}} / \mathrm{Ga}_{\mathrm{K}}$ concentration ratio between QDs and surrounding quantum well (QW) layers became well defined but less intense than that exhibited in the as-grown sample.

The obvious change in the $\mathrm{In}_{\mathrm{L}} / \mathrm{Ga}_{\mathrm{K}}$ concentration ratio between the as-grown and $800{ }^{\circ} \mathrm{C}$ annealed sample agrees with the theoretical change of the energy band structure, in which the
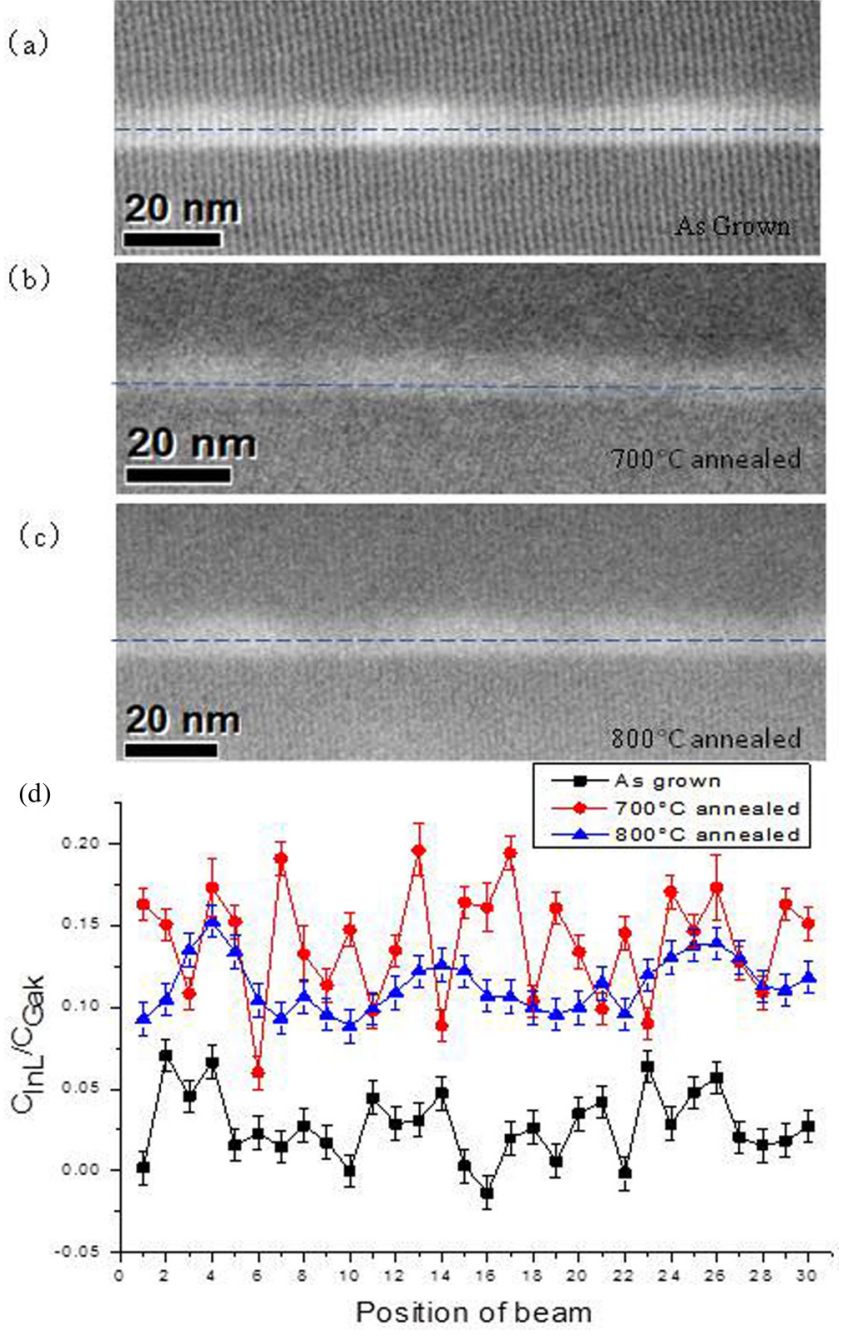

FIG. 9. HAADF-STEM images of (a) as-grown, (b) $700^{\circ} \mathrm{C}$ annealed, and (c) $800^{\circ} \mathrm{C}$ annealed InGaAs DWELLs; (d) compare the $\ln _{\llcorner} / \mathrm{Ga}_{K}$ concentration ratio of linescan region in as-grown, $700^{\circ} \mathrm{C}$ annealed, and $800^{\circ} \mathrm{C}$ annealed InGaAs DWELLs.

energy band of the InGaAs/InAs heteroepitaxial structure gradually changed at the interface between the QD and the surrounding QW after annealing. It further verifies that the confinement capacity of the energy band decreased after high-temperature RTA, increasing the rate of thermally assisted escape of carriers. Thus, less carriers are available to contribute to light emission and the PL intensity is decreased as shown in Fig. 3.

\section{CONCLUSION}

A PGA study has been carried out for an InAs/InGaAs/GaAs DWELL structure monolithically grown on a Si substrate. Firstly, compared to the DWELL structure directly grown on GaAs, the 
thermal annealing process induces a distinct difference in the tuning of the emission properties. Improvement in the emission intensity has been observed at $700{ }^{\circ} \mathrm{C}$ in the GaAs substrate sample. However, due to the stress induced by thermal annealing, the generation of deep levels limits the emission enhancement in a very narrow temperature window. Nevertheless, the simple and costeffective rapid thermal annealing treatment can improve the optical properties of the samples while preserving the emission wavelength of the DWELL structures grown on Si.

TEM imaging of the DWELL structures grown on Si substrates revealed that the PGA improved the uniformity of QDs and the size of QDs increased, in accordance with a red shift and the decrease of the FWHM in the PL emission spectrum. Finally, EDX linescan provided a semi-quantitative analysis of the composition change of DWELL grown on $\mathrm{Si}$ in the as-grown, $700{ }^{\circ} \mathrm{C}$ annealed, and $800{ }^{\circ} \mathrm{C}$ annealed samples. The change in the $\mathrm{In}_{\mathrm{L}} / \mathrm{Ga}_{\mathrm{K}}$ concentration ratio became gradual between QDs and surrounding materials after $800^{\circ} \mathrm{C}$ annealing. The effect of post-growth rapid thermal annealing on the DWELL structures grown on $\mathrm{Si}$ substrates has been studied. The analysis of the optical properties, morphological evolution, and compositional change of the QDs as a function of annealing temperature showed good agreement.

\section{ACKNOWLEDGMENTS}

This work was supported by the Engineering and Physical Sciences Research Council (EPSRC) (No. EP/P006973/1), Royal Academy of Engineering (No. RF201617/16/28), the Natural Science Foundation of China (No. 51872008), and Beijing Natural Science Foundation (No. Z180014). S. Chen acknowledges the Royal Academy of Engineering for funding his Research Fellowship.

\section{REFERENCES}

${ }^{1}$ Z. Zhang and R. A. Hogg, "Post-growth intermixing of GaAs based quantum dot devices," in Quantum Dot Devices (Springer, New York, 2012), pp. 109-130. ${ }^{2}$ D. Sengupta, V. Jandhyala, S. Kim, W. Fang, J. Malin, P. Apostolakis, K. C. Hseih, Y. C. Chang, S. L. Chuang, S. Bandara, and S. Gunapala, "Redshifting and broadening of quantum-well infrared photodetector's response via impurity-free vacancy disordering," IEEE J. Sel. Top. Quantum Electron. 4(4), 746-757 (1998).

${ }^{3}$ Z. Y. Zhang, Q. Jiang, I. J. Luxmoore, and R. A. Hogg, "A p-type-doped quantum dot superluminescent LED with broadband and flat-topped emission spectra obtained by post-growth intermixing under a GaAs proximity cap," Nanotechnology 20(5), 055204 (2009).

${ }^{4} \mathrm{~J}$. Tatebayashi et al., "InAs/GaAs self-assembled quantum-dot lasers grown by metalorganic chemical vapor deposition-Effects of post-growth annealing on stacked InAs quantum dots," Appl. Phys. Lett. 85, 1024 (2004).

${ }^{5} \mathrm{Q}$. Li et al., "Development of modulation p-doped $1310 \mathrm{~nm} \mathrm{InAs/GaAs}$ quantum Dot laser materials and ultrashort cavity fabry-perot and distributedfeedback laser diodes," ACS Photonics 5(3), 1084-1093 (2018).

${ }^{6}$ A. O. Kosogov, P. Werner, U. Gösele, N. N. Ledentsov, D. Bimberg, V. M. Ustinov, A. Y. Egorov, A. E. Zhukov, P. S. Kop'eV, N. A. Bert, and Z. I. Alferov, "Structural and optical properties of InAs-GaAs quantum dots subjected to high temperature annealing," Appl. Phys. Lett. 69(20), 3072-3074 (1996).

${ }^{7}$ G. Zhang and M. Pessa, "Thermal processing of strained-layer InGaAs/GaAs quantum well interface," Appl. Surf. Sci. 75(1-4), 274-278 (1994).
${ }^{8}$ A. Babiński, J. Jasiński, R. Bożek, A. Szepielow, and J. M. Baranowski, "Rapid thermal annealing of InAs/GaAs quantum dots under a GaAs proximity cap," Appl. Phys. Lett. 79(16), 2576-2578 (2001).

${ }^{9}$ J. M. Garcia, G. Medeiros-Ribeiro, K. Schmidt, T. Ngo, J. L. Feng, A. Lorke, J. Kotthaus, and P. M. Petroff, "Intermixing and shape changes during the formation of InAs self-assembled quantum dots," Appl. Phys. Lett. 71(14), 2014-2016 (1997).

${ }^{10}$ S. J. Xu, X. C. Wang, S. J. Chua, C. H. Wang, W. J. Fan, J. Jiang, and X. G. Xie, "Effects of rapid thermal annealing on structure and luminescence of selfassembled InAs/GaAs quantum dots," Appl. Phys. Lett. 72(25), 3335-3337 (1998).

${ }^{11}$ S. Malik, C. Roberts, R. Murray, and M. Pate, "Tuning self-assembled InAs quantum dots by rapid thermal annealing," Appl. Phys. Lett. 71(14), 1987-1989 (1997).

${ }^{12}$ L. Fu, P. Lever, H. H. Tan, C. Jagadish, P. Reece, and M. Gal, "Study of intermixing in InGaAs/(Al) GaAs quantum well and quantum dot structures for optoelectronic/photonic integration," IEE Proc. Circ. Dev. Syst. 152(5), 491-496 (2005).

${ }^{13}$ D. G. Deppe and N. Holonyak, Jr., "Atom diffusion and impurity-induced layer disordering in quantum well III-V semiconductor heterostructures," J. Appl. Phys. 64(12), R93-R113 (1988).

${ }^{14}$ B. S. Ooi, K. McIlvaney, M. W. Street, A. S. Helmy, S. G. Ayling, A. C. Bryce, J. H. Marsh, and J. S. Roberts, "Selective quantum-well intermixing in GaAs-AlGaAs structures using impurity-free vacancy diffusion," IEEE J. Quant. Electron. 33(10), 1784-1793 (1997).

${ }^{15}$ J. H. Marsh, "Quantum well intermixing," Semicond. Sci. Technol. 8, 1136 (1993).

${ }^{16}$ H. S. Djie, Y. Wang, Y. H. Ding, D. N. Wang, J. C. Hwang, X. M. Fang, Y. Wu, J. M. Fastenau, A. W. Liu, G. T. Dang, and W. H. Chang, "Quantum dash intermixing," IEEE J. Sel. Top. Quantum Electron. 14(4), 1239-1249 (2008).

${ }^{17}$ C. K. Chia, S. J. Chua, J. R. Dong, and S. L. Teo, "Ultrawide band quantum dot light emitting device by postfabrication laser annealing," Appl. Phys. Lett. 90(6), 061101 (2007).

${ }^{18}$ S. Chen, M. Liao, M. Tang, J. Wu, M. Martin, T. Baron, A. Seeds, and H. Liu, "Electrically pumped continuous-wave $1.3 \mathrm{um}$ InAs/GaAs quantum dot lasers monolithically grown on on-axis $\mathrm{Si}$ (001) substrates," Opt. Express 25, 4632-4639 (2017).

${ }^{19}$ Y. Wang, S. Chen, Y. Yu, L. Zhou, L. Liu, C. Yang, and S. Yu, "Monolithic quantum-dot distributed feedback laser array on silicon," Optica 5(5), 528-533 (2018).

${ }^{20}$ M. Liao, S. Chen, J. Park, A. Seeds, and H. Liu, "III-V quantum-dot lasers monolithically grown on silicon," Semicond. Sci. Technol. 33, 123002 (2018).

${ }^{21}$ T. Wang, H. Liu, A. Lee, F. Pozzi, and A. Seeds, " $1.3 \mu \mathrm{m}$ InAs/GaAs quantumdot lasers monolithically grown on Si substrates," Opt. Express 19(12), 11381-11386 (2011).

${ }^{22}$ M. Liao, S. Chen, S. Huo, S. Chen, J. Wu, M. Tang, and H. Liu, "Monolithically integrated electrically pumped continuous-wave III-V quantum dot light sources on silicon," IEEE J. Sel. Top. Quantum Electron. 23(6), 1-10 (2017).

${ }^{23}$ I. M. Ross and T. Walther, "Configuring a $300 \mathrm{kV}$ cold field-emission gun for optimum analytical performance," J. Phys. Conf. Ser. 371, 012012 (2012).

${ }^{24}$ R. Leon, Y. Kim, C. Jagadish, M. Gal, J. Zou, and D. J. H. Cockayne, "Effects of interdiffusion on the luminescence of InGaAs/GaAs quantum dots," Appl. Phys. Lett. 69(13), 1888-1890 (1996).

${ }^{25}$ R. Leon, S. Fafard, P. G. Piva, S. Ruvimov, and Z. Liliental-Weber, "Tunable intersublevel transitions in self-forming semiconductor quantum dots," Phys. Rev. B 58(8), R4262 (1998).

${ }^{26}$ S. Fafard and C. N1 Allen, "Intermixing in quantum-dot ensembles with sharp adjustable shells," Appl. Phys. Lett. 75(16), 2374-2376 (1999).

${ }^{27} \mathrm{M}$. A. Cusack, P. R. Briddon, and M. Jaros, "Electronic structure of InAs/GaAs self-assembled quantum dots,” Phys. Rev. B 54(4), R2300 (1996). 
${ }^{28}$ J. Kim, L.-W. Wang, and A. Zunger, "Comparison of the electronic structure of InAs/GaAs pyramidal quantum dots with different facet orientations," Phys. Rev. B 57(16), R9408 (1998)

${ }^{29} \mathrm{H}$. Jiang and J. Singh, "Strain distribution and electronic spectra of InAs/GaAs self-assembled dots: An eight-band study," Phys. Rev. B 56(8), 4696 (1997).

${ }^{30}$ M. C. Parri, Y. Qiu, and T. Walther, "New pathways for improved quantification of energy-dispersive X-ray spectra of semiconductors with multiple X-ray lines from thin foils investigated in transmission electron microscopy," J. Microsc. 260(3), 427-441 (2015).

${ }^{31} \mathrm{~T}$. Walther and X. Wang, "Self-consistent method for quantifying indium content from X-ray spectra of thick compound semiconductor specimens in a transmission electron microscope," J. Microsc. 262(2), 151-156 (2015).

${ }^{32}$ J. S. Tsang, C. P. Lee, S. H. Lee, K. L. Tsai, C. M. Tsai, and J. C. Fan, "Compositional disordering of InGaAs/GaAs heterostructures by lowtemperature-grown GaAs layers,” J. Appl. Phys. 79(2), 664-670 (1996). 